\title{
Acute Suppuration of the Pancreatic Duct in a Patient with Tropical Pancreatitis
}

\author{
Liliane S. Deeb Jasmeet Bajaj Sandeep Bhargava \\ David Alcid C.S. Pitchumoni
}

Saint Peter's University Hospital, Drexel University College of Medicine,

New Brunswick, N.J., USA

\section{Key Words}

Tropical pancreatitis · Pancreatic duct suppuration · Klebsiella ornithinolytica

\begin{abstract}
Background/Aim: Pancreatic sepsis secondary to infected necrosis, pseudocyst, or pancreatic abscess is a well-known clinical entity. Acute suppuration of the pancreatic duct (ASPD) in the setting of chronic calcific pancreatitis and pancreatic ductal obstruction with septicemia is a rare complication that is seldom reported. It is our aim to report a case of ASPD with Klebsiella ornithinolytica, in the absence of pancreatic abscess or infected necrosis.
\end{abstract}

Case Report: A 46-year-old Asian-Indian man with chronic tropical pancreatitis who was admitted with recurrent epigastric pain that rapidly evolved into septic shock. A CT scan of abdomen revealed a dilated pancreatic duct with a large calculus. Broad-spectrum antibiotics, vasopressors and activated recombinant protein $C$ were initiated. Emergency ERCP showed the papilla of Vater spontaneously expelling pus. Probing and stenting was instantly performed until pus drainage ceased. Repeat CT scan confirmed the absence of pancreatic necrosis or fluid collection, and decreasing ductal dilatation. Dramatic clinical improvement was observed within 36 hours after intervention. Blood cultures grew Klebsiella ornithinolytica. The patient completed his antibiotic course and was discharged.

Conclusion: ASPD without pancreatic abscess or infected necrosis is an exceptional clinical entity that should be included in the differential diagnosis of pancreatic sepsis. A chronically diseased pancreas and diabetes may have predisposed to the uncommon pathogen. The presence of intraductal pancreatic stones obstructing outflow played a major role in promoting bacterial growth, suppuration and septicemia. Immediate drainage of the pancreatic duct with endoscopic intervention is critical and mandatory. 


\section{Introduction}

Pancreatic sepsis secondary to infected necrosis, pseudocyst, or contiguous pancreatic abscess is a well-known clinical entity complicating pancreatitis [1]. However, acute suppuration of the pancreatic duct (ASPD) in the setting of chronic pancreatitis and pancreatic ductal obstruction leading to septicemia is an extremely rare complication that is seldom reported. ASPD has been previously described in a few isolated case reports [24]. We report ASPD in the setting of obstructing intraductal stones leading to Klebsiella ornithinolytica septicemia in a patient with tropical calcific pancreatitis.

\section{Case Report}

A 46-year-old Asian-Indian man with no history of alcohol consumption was diagnosed to have tropical chronic pancreatitis in 2002 and was maintained on oral pancreatic enzyme therapy for exocrine pancreatic insufficiency. Three years later he developed non-insulin-dependent diabetes mellitus and was prescribed glucotrol (glipizide) $5 \mathrm{mg}$ daily. This patient presented to Saint Peter's University Hospital, New Brunswick, NJ, complaining of a steady midepigastric pain of 6 hours duration, dull in nature, radiating through his back and exacerbated by food intake. This pain was recurrent since his diagnosis with tropical pancreatitis, and often lasted between 1 and 3 days. In the interval phase, he remained completely well. For that he was hospitalized for pain control with narcotics. However, 2 days after discharge the patient returned complaining of continuing epigastric pain that gradually increased in intensity, associated with nausea and an episode of vomiting but no documented fever. A CT scan performed in the emergency department revealed fatty atrophy of both the pancreatic head and uncinate process. The pancreatic duct was filled with large calcifications with a possible obstruction at the level of the neck resulting in marked upstream ductal dilatation of $1.5 \mathrm{~cm}$ (fig. 1). He was again admitted for management of pain secondary to chronic pancreatitis.

Approximately 2 months prior to these events, the patient presented to a medical center in India with similar pain and fever for which he underwent a CT scan of the abdomen that revealed chronic atrophic calcific pancreatitis, with dilated pancreatic duct $(7 \mathrm{~mm})$ and intraductal calculi. An endoscopic retrograde cholangiopancreatography (ERCP) was performed that confirmed the above findings on pancreatography. He was then medically managed and discharged to return for removal of ductal stones once this acute exacerbation resolved fully.

On the second day of the current admission he developed fever $\left(102^{\circ} \mathrm{F}\right)$, chills and rigors and rapidly progressed to severe sepsis and septic shock within hours. Subsequently the patient was transferred to the medical intensive care unit and was intubated for hypoxemic respiratory failure. Clinical parameters were consistent with acute respiratory distress syndrome and multiorgan failure. At that point, his management included the administration of broad-spectrum antibiotics, vasopressor support and activated recombinant human protein $\mathrm{C}$. An ultrasound of the abdomen was done showing a markedly dilated pancreatic duct $(1.9 \mathrm{~cm})$ with an $8.7 \times 7.6 \mathrm{~mm}$ calculus within the duct. The pancreas was diffusely echogenic. The common bile duct was prominent with no obvious obstructing calculi.

A bedside emergency ERCP was performed in the intensive care unit setting using the Olympus TJF160 side viewing video duodenoscope. The major papilla of Vater was visualized spontaneously expelling frank pus (fig. 2). There was no evidence of papillitis, tumor or previous sphincterotomy. This orifice was then probed with the cannula tip prompting the evacuation of more than $5 \mathrm{ml}$ of yellow pus. Because of its viscosity the fluid could not be aspirated through the cannula for culture. A cholangiogram was obtained revealing a normal biliary tree without stones. The pancreatogram showed marked dilatation of the main pancreatic duct, with a single distal calculus. A guide wire was introduced into the pancreatic duct and a total of approximately $20 \mathrm{ml}$ of pus was then evacuated during duct manipulation. After achieving selective cannulation, a 5-cm-long $5 \mathrm{~F}$ stent was placed into the pancreatic duct but it failed to show any more pus draining so decision was taken to pull it out. At this point it was assumed that the patient had an ASPD possibly secondary to the presence of the intraductal calculus. A reevaluation of the pancreas with a contrast enhanced CT scan of the abdomen was performed on the following day. It showed inflammatory changes within the fat surrounding the body and tail of the pancreas consistent with edema. Compared to the previous study the dilatation of the pancreatic duct had diminished with the calculus showing distal migration towards the sphincter of Oddi. There was no evidence of pancreatic necrosis or fluid collection (fig. 3 ). Bilateral moderate pleural effusions were present. 

improvement with stabilization of his hemodynamic parameters. Blood cultures grew Klebsiella ornithinolytica sensitive to the antibiotics given. He was extubated, transferred from the intensive care unit, completed his antibiotic course and was discharged home 11 days after admission. Follow-up examinations at 1 month and 3 months after discharge revealed no further complications.

\section{Discussion}

The above patient presented with septicemia, shock and acute purulent discharge from the pancreatic duct without evidence of any infected necrotic tissue or a coexisting pancreatic abscess. ASPD without concurrent pancreatic abscess or infected necrosis remains an exceptional clinical entity [3].

The two major groups of infective complications occurring after an attack of acute pancreatitis are infections associated with necrotic tissue (infected necrosis) and infections arising without tissue necrosis (pancreatic abscess). In the second category purulent collections walled off around the pancreas or in surrounding tissues can occur. A subgroup of pancreatic abscesses is the group of patients with infected pseudocysts. The distinction between infected necrosis and pancreatic abscess is usually clear-cut [5]. The trivial and seldom reported entity is the one we are highlighting in this case report. Further, this is a complication of chronic pancreatitis with dilated and obstructed duct system and perhaps precipitated by ductal instrumentation [5].

Our patient had multiple preexisting risk factors for the development of ASPD. In a normal pancreas, bacteriostatic and bactericidal substances elaborated in pancreatic secretions provide resistance to bacterial infections. Hence a chronically diseased pancreas may be much more susceptible to infection because these same antibacterial substances are significantly impaired [6,7]. Moreover diabetes mellitus predisposes patients to a variety of uncommon infections and may have played an additive role in this patient's infection with Klebsiella ornithinolytica. This bacterium is usually contracted by oral route [8] and one can only speculate that during previous instrumentation of the ampulla of Vater, duodenal contents contaminated with this unusual organism may have refluxed into the pancreatic duct resulting effectively in seeding of the pancreatic juice. However, the presence of bacteria in the pancreatic duct is not by itself sufficient to cause suppuration [7]. The presence of intraductal pancreatic stones obstructing the outflow of contaminated pancreatic secretions may have played a major role in this patient's ASPD. Bacterial overgrowth distal to the obstruction ensued, leading to actual suppuration and secondary septicemia. This would be analogous to calculus obstruction of the biliary tree resulting in acute suppuration and ascending cholangitis.

This case proposes the concept that ASPD is a complex process involving a chronically damaged pancreas, reflux of contaminated duodenal contents into the pancreatic duct, pancreatic outflow obstruction and subsequent bacterial infection or 'infectious' pancreatitis. ASPD is an exceptional clinical entity that should be included in the differential diagnosis of pancreatic sepsis. Immediate drainage of the pancreatic duct with endoscopic intervention is critical and mandatory. 
Fig. 1. A CT image with contrast demonstrates a marked pancreatic ductal dilatation (black arrow) with large intraductal calcifications (white arrow).

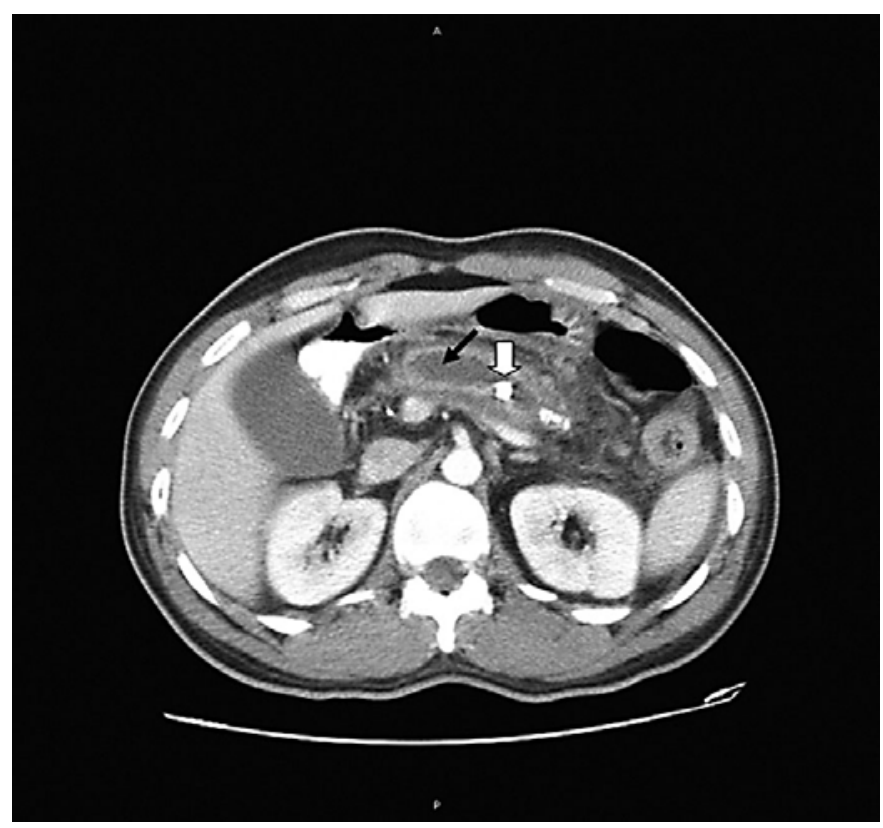

Fig. 2. An endoscopic retrograde view showing frank pus expelling spontaneously through the major papilla of Vater.

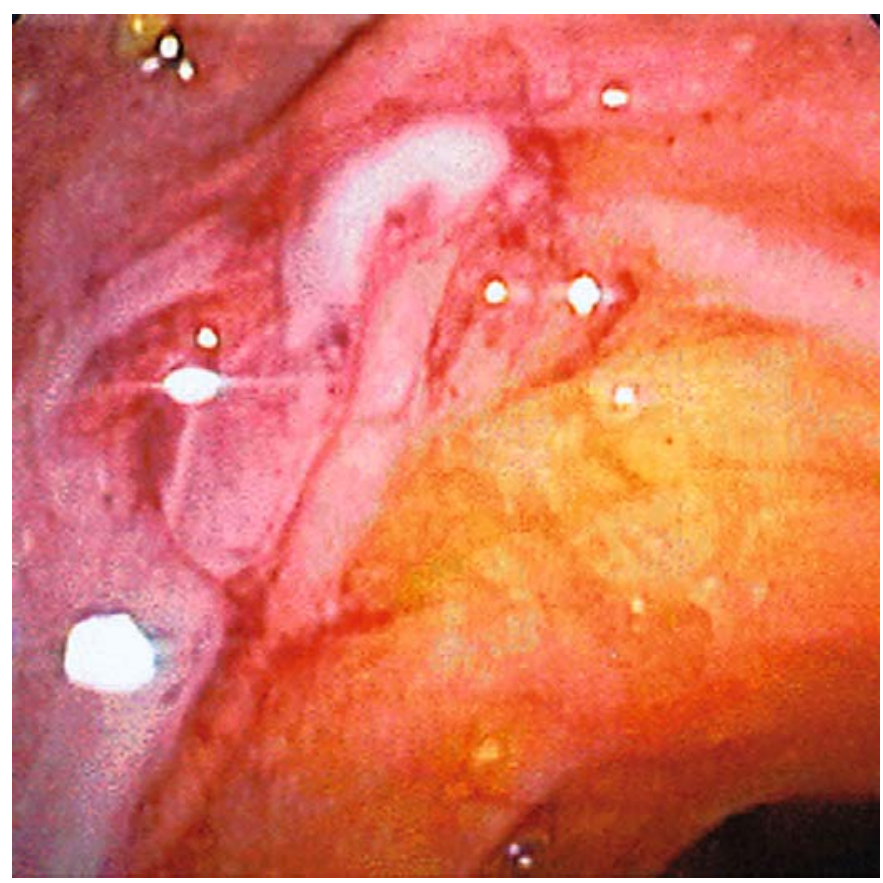


Fig. 3. A follow-up CT image of the pancreas showing distal migration of pancreatic stones (white arrow) with diminished ductal dilatation (black arrow).

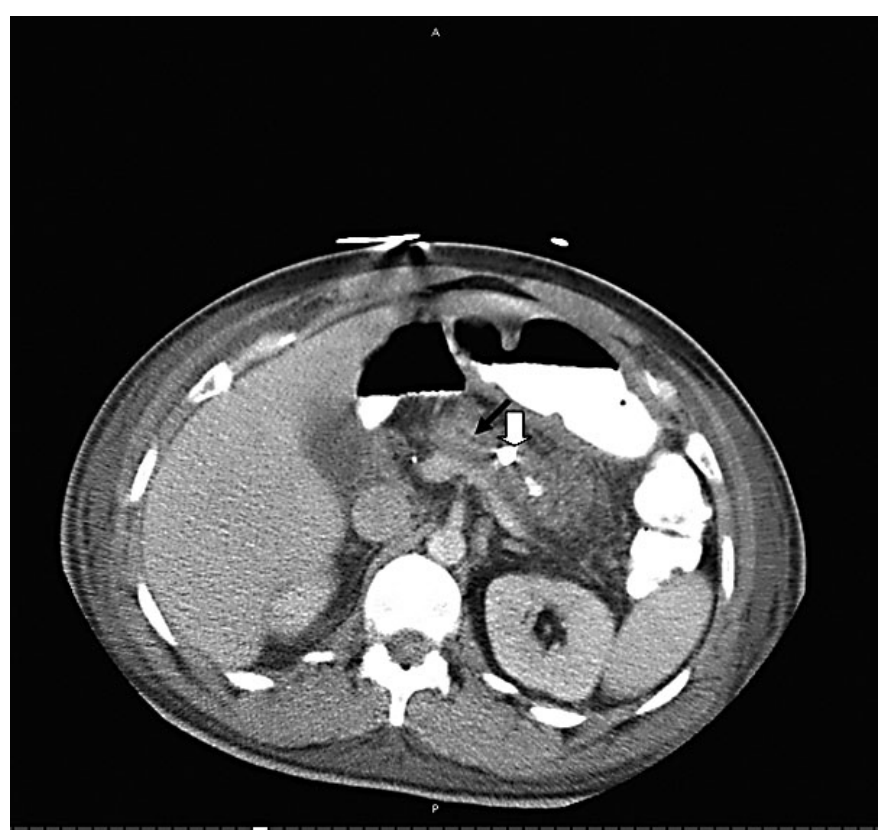




\section{References}

1 Hookey LC, Debroux S, Delhaye M, Arvanitakis M, Le Moine O, Devière J: Endoscopic drainage of pancreatic-fluid collections in 116 patients: A comparison of etiologies, drainage techniques, and outcomes. Gastrointest Endosc 2006;63:635-643.

2 Weinman DS: Acute suppuration of the pancreatic duct. Gastrointest Endosc 1995;41:268-270.

-3 Tajima Y, Kuroki T, Susumu S, Tsutsumi R, Kitasato A, Adachi T, Mishima T, Kanematsu T: Acute suppuration of the pancreatic duct associated with pancreatic ductal obstruction due to pancreas carcinoma. Pancreas 2006;33:195197.

-4 Pai CG, Alvares JF: Endoscopic pancreatic-stent placement and sphincterotomy for relief of pain in tropical pancreatitis: Results of a 1-year follow-up. Gastrointest Endosc 2007;66:70-75.

5 Geoffrey G: Epidemiology and pathology of pancreatic abscess; in Bradley E (ed): Acute Pancreatitis, Diagnosis and Therapy. New York, Raven Press, 1994, pp 153-154.

-6 Marotta F, Tajiri H, Li ZL, Barreto R, Belline O, Barbi G: Pure pancreatic juice from patients with chronic pancreatitis has an impaired antibacterial activity. Int J Pancreatol 1997;22:215-220.

-7 Kruszewska D, Ljungh A, Hynes SO, Pierzynowski SG: Effect of the antibacterial activity of pig pancreatic juice on human multiresistant bacteria. Pancreas 2004;28:191-199.

-8 Henriques IS, Fonseca S, Alves A, Saavedra MJ, Correia A: Occurrence and diversity of integrons and beta-lactamase genes among ampicillin-resistant isolates from estuarine waters. Res Microbiol 2006;157:938-947. 